\title{
Oblique Scattering by a Pair of Conducting Half Planes: TM Case
}

\author{
Jong-Won Yu and Noh-Hoon Myung
}

\begin{abstract}
An exact series solution for the oblique scattering by a pair of infinitely long parallel edges of perfectly conducting half planes is formulated for a TM plane wave using the modematching technique (MMT). The scattered and guided fields are represented in terms of an infinite series of radial waveguide modes. By applying the appropriate boundary conditions, the coefficients of the scattered field are obtained. The diffraction coefficient of double edges is subsequently derived from the scattered field.
\end{abstract}

Index Terms - Electromagnetic diffraction, mode-matching technique.

\section{INTRODUCTION}

$\mathbf{E}$ LECTROMAGNETIC diffraction by perfectly conducting double-edge structures has been a subject of intensive research. An asymptotic solution to the high-frequency diffraction by a single edge may be obtained by employing the geometrical theory of diffraction (GTD) [1] and its uniform extension (UTD) [2]. Since they are ray-based theories, they cannot treat double-edge geometries accurately when the second wedge is illuminated by a transition region field, where the diffracted field shows rapid spatial variation in the vicinity of the edge. During the past decade, a variety of approaches have been taken to overcome these limitations.

Tiberio and Kouyoumjian [3], [4] employed an extended spectral theory of diffraction (ESTD) to investigate the diffraction by the edges within the transition region of singly diffracted fields. Their solution, however, is restricted to the case where either the source or receiver is on the same plane with the two edges. Michaeli [5], [6] examined the farfield scattering by a pair of parallel edges using the analysis, which is based on the physical theory of diffraction (PTD). It is valid when the width of the double wedge gap is small compared to the source and observer distance. Schneider and Luebbers [7] also developed a uniform double-edge diffraction coefficient by applying ESTD, which correctly compensates for the discontinuities of the single-edge diffracted field. Their expression involves a double integral over an infinite domain, which can be represented in terms of a convergent series of Bessel functions close to the shadow boundaries. However, it cannot be tabulated easily.

Most of the previous work deals with scattering from a double wedge when the plane of the incidence is perpendicular

Manuscript received September 22, 1997; revised June 14, 1999.

The authors are with the Department of Electrical Engineering, Korea Advanced Institute of Science and Technology (KAIST), Taejon, 305-701 Korea.

Publisher Item Identifier S 0018-926X(99)09947-0.

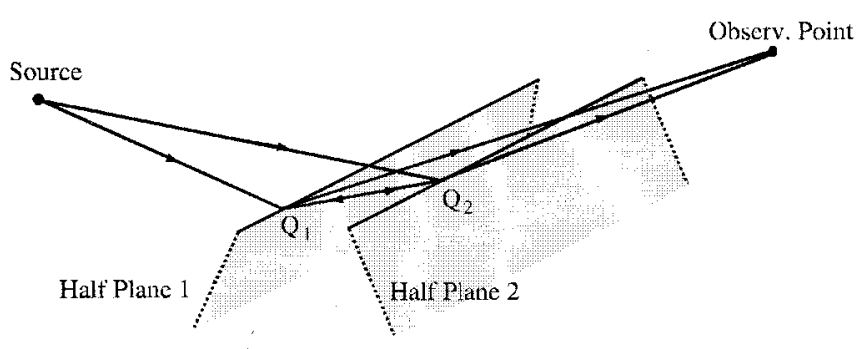

(a)

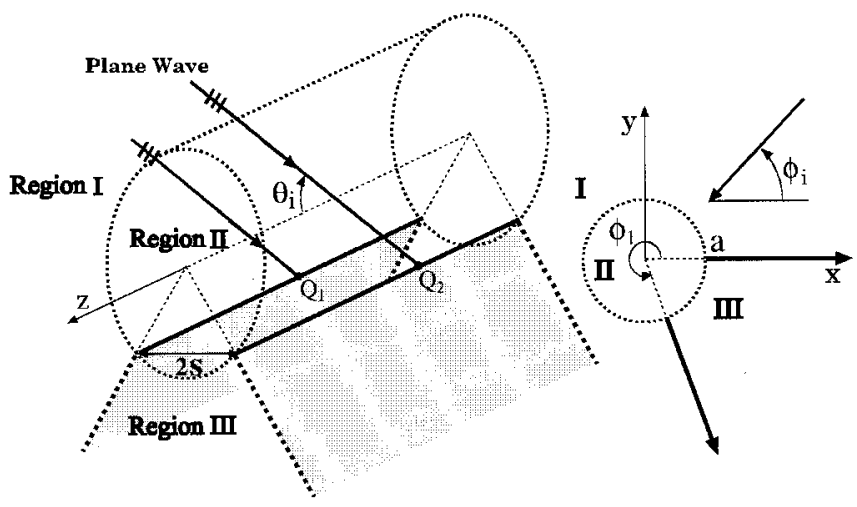

(b)

Fig. 1. (a) Scattering by a pair of perfectly conducting half planes. (b) Analytical model of the problem.

to the wedge axis. Hence, the scattering behavior is not well understood when the plane of incidence is at an arbitrary angle with respect to the wedge axis (three-dimensional oblique incidence case). Ivrissimtzis and Marhefka [8], [9] developed a near-zone solution for the doubly diffracted field from a pair of coplanar skewed edges.

In this paper, a simple series solution for the oblique scattering by a pair of parallel edges of perfectly conducting half planes (shown in Fig. 1) is investigated. The diffraction coefficient of the double edges is subsequently derived and formulated from the scattered field.

\section{THEORETICAL FORMULATION}

Consider a $T M_{z}$ plane wave at $\phi=\phi_{i}$ and $\theta=\theta_{i}$ illuminating a pair of infinitely long parallel edges of conducting half planes, as shown in Fig. 1. Throughout the paper, the $e^{j \omega t}$ time-harmonic factor is assumed and suppressed. A fictitious circle of radius $a$ is set up to connect the parallel edges of two half planes, as shown in Fig. 1(b). The center of the fictitious circle is the origin of coordinate, which is also the intersecting 


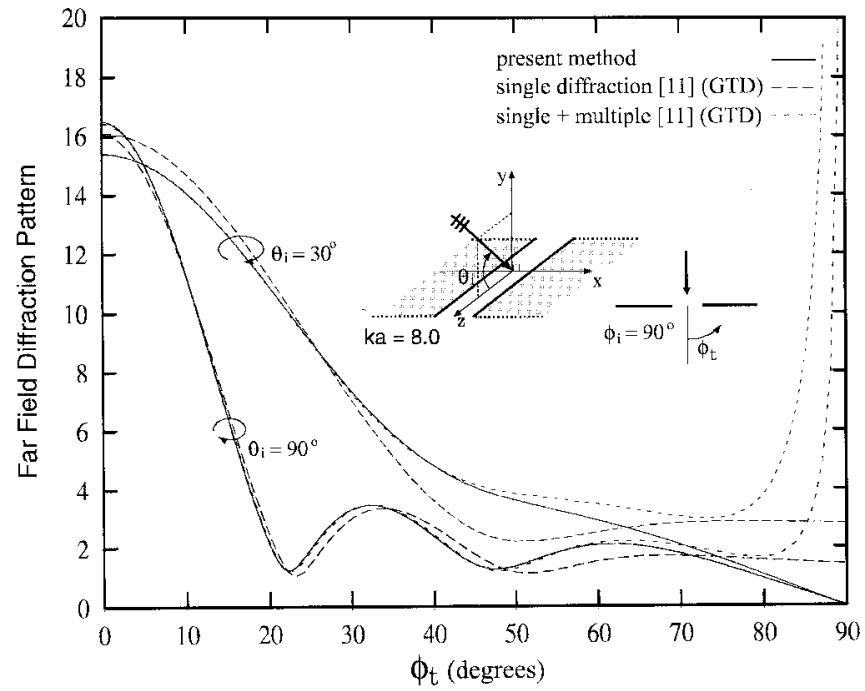

Fig. 2. Far-field diffraction pattern for a slit of $k a=8$ versus $\phi_{t}$ when $\phi_{i}=90^{\circ}$.

point of extended lines of two side edges, as shown in the figure. The two half planes are located in a radial direction with respect to the center of the cylinder and $\operatorname{span} \phi=0$ and $\phi_{1}$. As shown in Fig. 1(b), Regions I, II, and III denote the region of cylinder upper, inside, and lower sectors, respectively.

The total electric field in Region I $\left(\rho>a, 0<\phi<\phi_{1}\right)$ which satisfies the boundary condition $E_{\tan }=0$ on the wedge and the radiation condition, is given by

$$
E_{z}^{I}(\rho, \phi)=F\left(\theta_{i}\right) \sum_{p=1}^{\infty}\left\{s_{p} j^{\mu} J_{\mu}(\kappa \rho)+B_{p} H_{\mu}^{(2)}(\kappa \rho)\right\} \sin \mu \phi
$$

where

$$
\begin{aligned}
s_{p} & =\frac{4 \pi}{\phi_{1}} \sin \mu \phi_{i} \\
\mu & =\frac{p \pi}{\phi_{1}}, \quad p=1,2,3 \cdots \\
F\left(\theta_{i}\right) & =\sin \theta_{i} e^{j k_{o} z \cos \theta_{i}} \\
\kappa & =k_{o} \sin \theta_{i}
\end{aligned}
$$

and $J_{\mu}(\cdots)$ and $H_{\mu}^{(2)}(\cdots)$ are the Bessel function, the first kind of $\mu$ th order, and the Hankel function, the second kind of $\mu$ th order, respectively. The first term in (1) is the total field around an infinite wedge formed by the intersection of the two planes and the second term is a perturbation effect by the two edges.

In Regions II $(\rho<a)$ and III $\left(\rho>a, \phi_{1}<\phi<2 \pi\right)$, the total electric field may be represented as a summation of radial waveguide modes, i.e.,

$$
\begin{aligned}
E_{z}^{I I}(\rho, \phi) & =F\left(\theta_{i}\right) \sum_{n=-\infty}^{\infty} A_{n} J_{n}(\kappa \rho) e^{j n \phi} \\
E_{z}^{I I I}(\rho, \phi) & =F\left(\theta_{i}\right) \sum_{q=1}^{\infty} C_{q} H_{\nu}^{(2)}(\kappa \rho) \sin \nu\left(\phi-\phi_{1}\right)
\end{aligned}
$$

respectively, where $\nu=q \pi /\left(2 \pi-\phi_{1}\right), q=1,2,3 \cdots$.

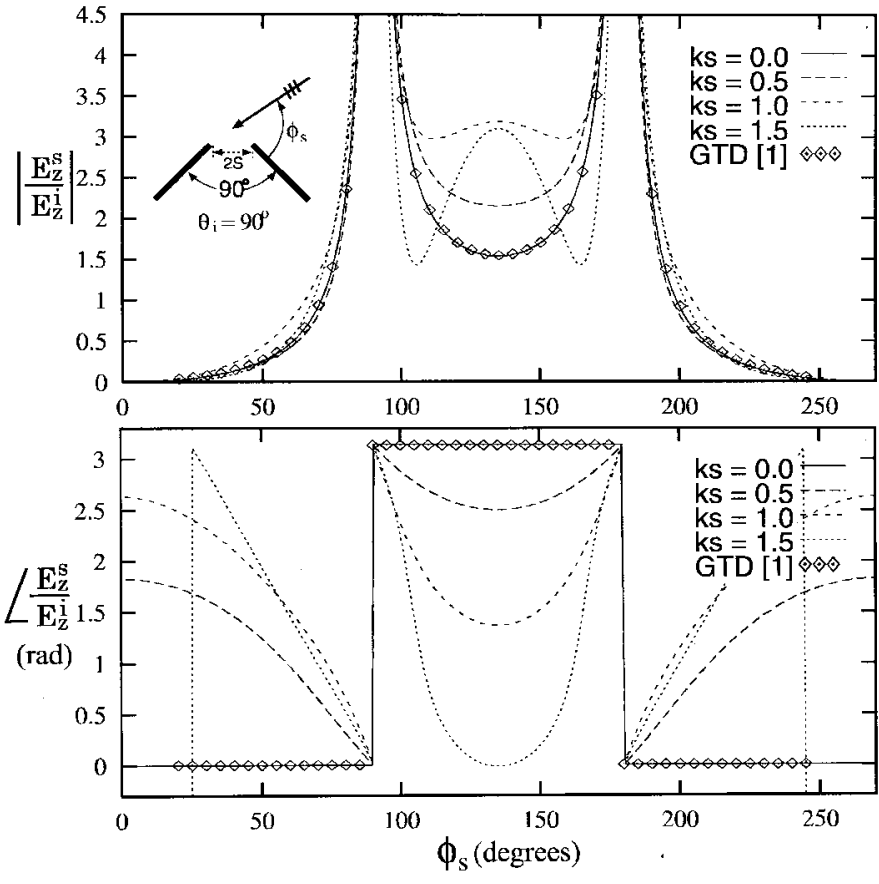

Fig. 3. Normalized backscattered field of $E_{z}^{s} / E_{z}^{i}$ versus $\phi_{s}$ when $\phi_{1}=270^{\circ}$ and $\theta_{i}=90^{\circ}$.

To determine the unknown coefficients $A_{n}, B_{p}$, and $C_{q}$, it is necessary to match the tangential $E$ - and $H$-fields at $\rho=a$. First, the tangential $E$-field continuity at $\rho=a$ yields

$$
\begin{aligned}
\sum_{n=-\infty}^{\infty} & A_{n} J_{n}(\kappa a) e^{j n \phi} \\
= & U_{I} \sum_{p=1}^{\infty}\left\{s_{p} j^{\mu} J_{\mu}(\kappa a)+B_{p} H_{\mu}^{(2)}(\kappa a)\right\} \sin \mu \phi \\
& +U_{I I} \sum_{q=1}^{\infty} C_{q} H_{\nu}^{(2)}(\kappa a) \sin \nu\left(\phi-\phi_{1}\right)
\end{aligned}
$$

where $U_{I(I I)}=1$ for $0<\phi<\phi_{1}\left(\phi_{1}<\phi<2 \pi\right)$ and zero elsewhere. In (4), applying the orthogonality condition of exponential function with respect to $\phi$ from zero to $2 \pi$ gives

$$
\begin{aligned}
2 \pi A_{k} J_{k}(\kappa a)= & \sum_{p=1}^{\infty}\left\{s_{p} j^{\mu} J_{\mu}(\kappa a)+B_{p} H_{\mu}^{(2)}(\kappa a)\right\} F_{-k \mu} \\
& +\sum_{q=1}^{\infty} C_{q} H_{\nu}^{(2)}(\kappa a) G_{-k \nu}
\end{aligned}
$$

where

$$
\begin{aligned}
F_{-k \mu} & =\int_{0}^{\phi_{1}} e^{-j k \phi} \sin \mu \phi d \phi \\
G_{-k \nu} & =\int_{\phi_{1}}^{2 \pi} e^{-j k \phi} \sin \nu\left(\phi-\phi_{1}\right) d \phi .
\end{aligned}
$$




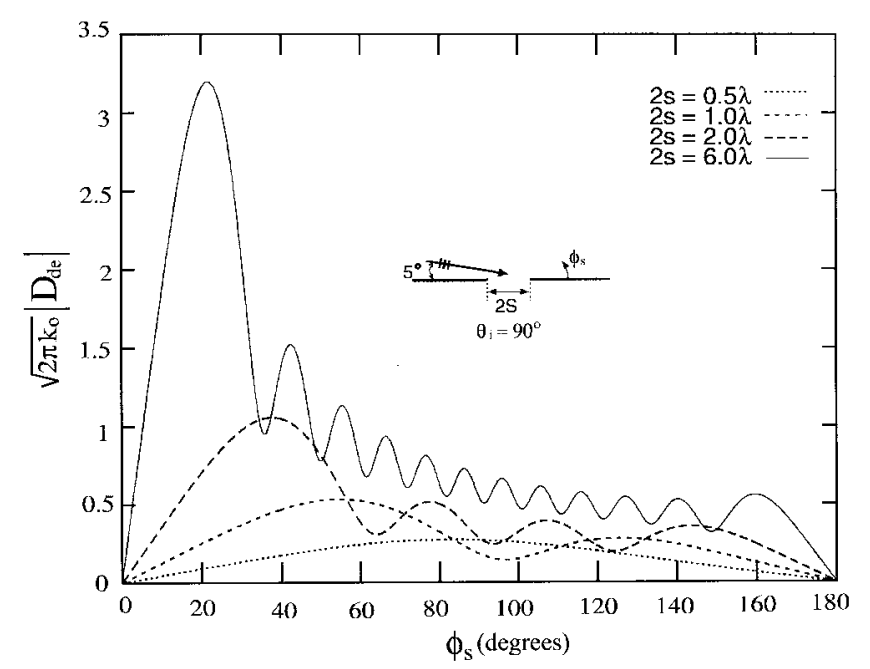

Fig. 4. Diffraction coefficient $\left(D_{d e}\right)$ versus $\phi_{s}$ as a function of $s$ for a slit at grazing incidence $\phi_{i}=175^{\circ}$.

Next, the tangential $H$-field continuity at $\rho=a$ also gives

$$
\begin{aligned}
\sum_{n=-\infty}^{\infty} & A_{n} J_{n}^{\prime}(\kappa a) e^{j n \phi} \\
= & \begin{cases}\sum_{p=1}^{\infty}\left\{s_{p} j^{\mu} J_{\mu}^{\prime}(\kappa a)+B_{p} H_{\mu}^{(2)^{\prime}}(\kappa a)\right\} \sin \mu \phi \\
0<\phi<\phi_{1} \\
\sum_{q=1}^{\infty} C_{q} H_{\nu}^{(2)^{\prime}}(\kappa a) \sin \nu\left(\phi-\phi_{1}\right) \\
\phi_{1}<\phi<2 \pi .\end{cases}
\end{aligned}
$$

Applying orthogonality conditions of the sine function to (6) with respect to $\phi$ from zero to $\phi_{1}$ and from $\phi_{1}$ to $2 \pi$ yields the following:

$$
\begin{aligned}
& B_{p} H_{\mu}^{(2)^{\prime}}(\kappa a)=\frac{2}{\phi_{1}} \sum_{k=-\infty}^{\infty} A_{k} J_{k}^{\prime}(\kappa a) F_{\mu k}-s_{p} j^{\mu} J_{\mu}^{\prime}(\kappa a) \\
& C_{q} H_{\nu}^{(2)^{\prime}}(\kappa a)=\frac{2}{2 \pi-\phi_{1}} \sum_{k=-\infty}^{\infty} A_{k} J_{k}^{\prime}(\kappa a) G_{\nu k}
\end{aligned}
$$

where

$$
\begin{aligned}
F_{\mu k} & =\int_{0}^{\phi_{1}} e^{j k \phi} \sin \mu \phi d \phi \\
G_{\nu k} & =\int_{\phi_{1}}^{2 \pi} e^{j k \phi} \sin \nu\left(\phi-\phi_{1}\right) d \phi
\end{aligned}
$$

In order to determine the coefficient $A_{n}$, (7) and (8) are substituted into (5). By applying the Wronskian formula

$$
J_{n}(x) H_{n}^{(2)^{\prime}}(x)-J_{n}^{\prime}(x) H_{n}^{(2)}(x)=-\frac{2 j}{\pi x}
$$

the following can be obtained:

$$
\begin{gathered}
\sum_{n=-\infty}^{\infty} A_{n} J_{n}(\kappa a)\left[\delta_{k n}-\frac{J_{n}^{\prime}(\kappa a)}{J_{n}(\kappa a)} I_{k n}\right] \\
=-\frac{j}{\pi^{2} \kappa a} \sum_{p=1}^{\infty} \frac{s_{p} j^{\mu}}{H_{\mu}^{(2)^{\prime}}(\kappa a)} F_{-k \mu}
\end{gathered}
$$

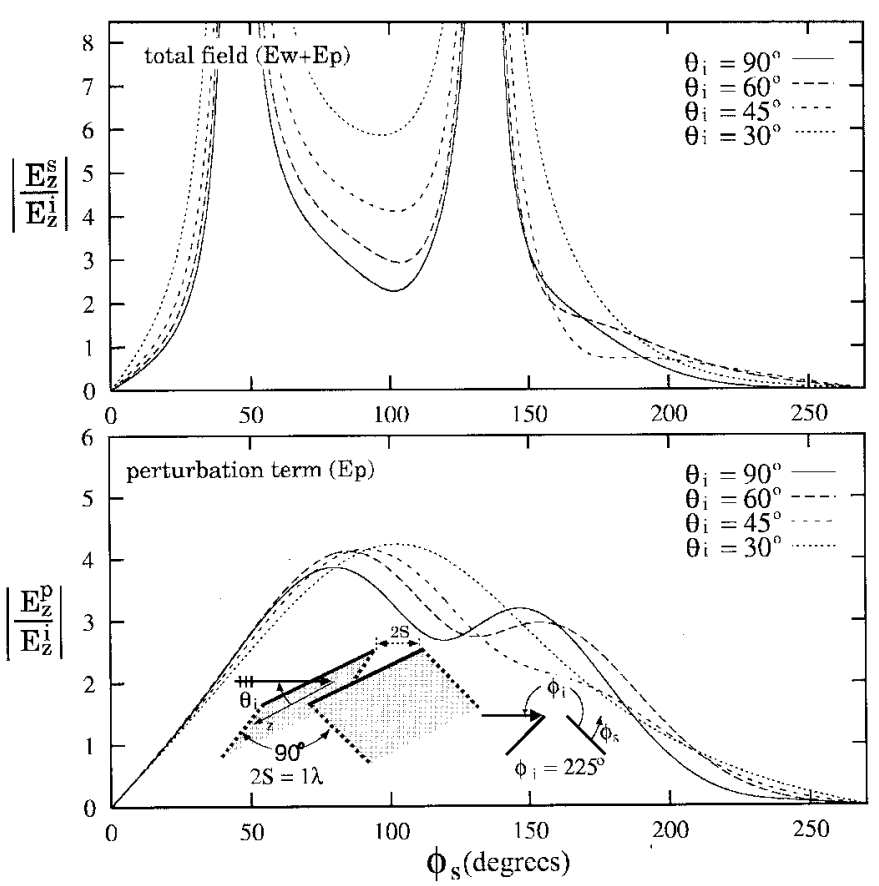

Fig. 5. Normalized scattered field pattern for the two half planes of $\phi_{1}=270^{\circ}, \phi_{i}=225^{\circ}$ and $2 s=1 \lambda$ in the case of four different oblique incidence $\left(\theta_{i}=30^{\circ}, 45^{\circ}, 60^{\circ}\right.$, and $\left.90^{\circ}\right)$.

where $\delta_{k n}$ is the Kronecker's delta and

$$
\begin{aligned}
I_{k n}=\frac{1}{\pi}\left(\sum_{p=1}^{\infty} \frac{F_{n \mu} F_{-k \mu}}{\phi_{1}} \frac{H_{\mu}^{(2)}(\kappa a)}{H_{\mu}^{(2)^{\prime}}(\kappa a)}\right. \\
\left.\quad+\sum_{q=1}^{\infty} \frac{G_{n \nu} G_{-k \nu}}{2 \pi-\phi_{1}} \frac{H_{\nu}^{(2)}(\kappa a)}{H_{\nu}^{(2)^{\prime}}(\kappa a)}\right) .
\end{aligned}
$$

Equation (9) can then be solved numerically to obtain $A_{n}$. The infinite series involved in the solution is convergent and this makes it possible to truncate it after a certain number of terms. Once $A_{n}$ is determined, $B_{p}$ and $C_{q}$ can subsequently be calculated from (7) and (8).

To obtain the scattered field for a $T M_{z}$ plane wave, the asymptotic expansion of the Hankel function for a large argument is employed together with the well-known approximation for the field diffracted by a sharp wedge [11]. The scattered field may be expressed as

$$
\left.\begin{array}{rl}
\frac{E_{z}^{s}}{E_{z}^{i}} \sim \frac{e^{-j(k \rho+\pi / 4)}}{\sqrt{2 \pi k \rho} \sin \theta_{i}}\left\{\frac{\sin (\pi / n)}{n}\right. & \\
\cdot & {\left[\frac{1}{\cos (\pi / n)-\cos \left(\left(\phi-\phi_{i}\right) / n\right)}\right.} \\
& \left.-\frac{1}{\cos (\pi / n)-\cos \left(\left(\phi+\phi_{i}\right) / n\right)}\right] \\
& +2 j \sum_{p=1}^{\infty} j^{\mu} B_{p} \sin \mu \phi
\end{array}\right\}
$$

where $n=\phi_{1} / \pi$. In (11), the first term is the field by a sharp wedge $\left(E_{z}^{w} / E_{z}^{i}\right)$ formed by extending the semi-infinite half planes and the second term represents a perturbation 

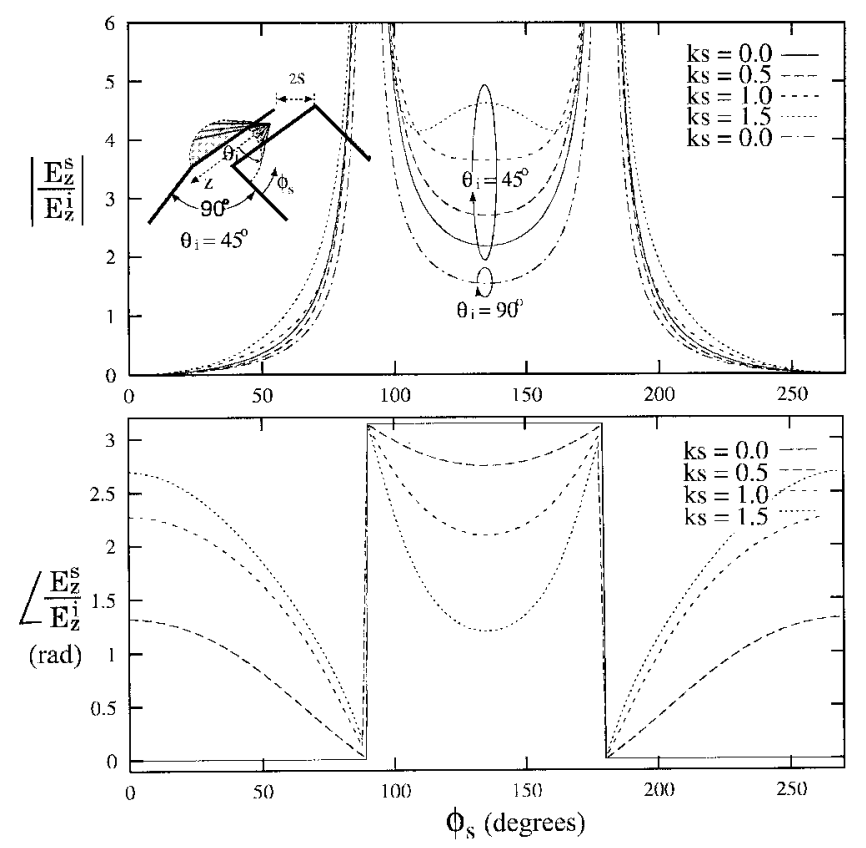

Fig. 6. Normalized backscattered field of $E_{z}^{s} / E_{z}^{i}$ versus $\phi$ for different gaps between two conducting half planes of $\phi_{1}=270^{\circ}$ in the case of oblique incidence $\left(\theta_{i}=45^{\circ}\right)$.

term $\left(E_{z}^{p} / E_{z}^{i}\right)$ for the double edges. Furthermore, the field of perturbation term may be related with incident field $E_{z}^{i}$ as follows:

$$
E_{z}^{p}=E_{z}^{i} D_{d e}\left(k a, \phi_{o}, \phi, \phi_{i}\right) \frac{e^{-j k \rho}}{\sqrt{\rho}}
$$

where

$$
D_{d e}\left(k a, \phi_{o}, \phi, \phi_{i}\right)=\sqrt{\frac{2}{\pi k}} \frac{e^{j \pi / 4}}{\sin \theta_{i}} \sum_{p=1}^{\infty} j^{\mu} B_{p} \sin \mu \phi
$$

and $D_{d e}$ is a diffraction coefficient of the double edges.

\section{NUMERICAL RESULTS}

In order to check the accuracy of present method developed in this paper, a slit $\left(\phi_{1}=180^{\circ}\right)$ is chosen for calculations as a special case of the double edges structure. Fig. 2 shows the far-field diffraction pattern of a slit with $k a=8.0$ versus $\phi_{t}$ at $\phi_{i}=90^{\circ}, \theta_{i}=30^{\circ}$, and $90^{\circ}$. The angle $\phi_{t}$ ranges between $0^{\circ}$ and $90^{\circ}$, corresponding to the region behind the slit. The number of modes used in computaions is $n=p=q=15$. As shown in the figure, the result obtained by the present method agrees well with GTD solutions [10] for $\phi_{t}<50^{\circ}$. It is noted that the GTD diffracted field shows singular behaviors around $\phi_{t}=90^{\circ}$. Fig. 3 shows the normalized backscattered field of $E_{z}^{s} / E_{z}^{i}$ versus $\phi_{s}$ for different gaps between two parallel edges of conducting half planes of $\phi_{1}=270^{\circ}$ and $\theta_{i}=90^{\circ}$. It is noted that the numerical data for $k s=0$ case agrees well with the $90^{\circ}$ sharp wedge backscattered field pattern. An increase in $k s$ causes a large variation in the pattern level at $90^{\circ}<\phi_{s}<180^{\circ}$ and a small variation at $\phi_{s}<90^{\circ}$ and $\phi_{s}>180^{\circ}$. Phase data presented in radians are continuous except for a step of $\pi$ radians at $\phi_{s}=90^{\circ}$ and $180^{\circ}$, which is originated by the singular behavior of the asymptotic result for the sharp wedge. Magnitude of the diffraction coefficient $D_{d e}$ versus $\phi_{s}$ is shown in Fig. 4 as a function of $s$ at grazing incidence of $\phi_{i}=175^{\circ}$ and $\theta_{i}=90^{\circ}$. As $s$ increases, pattern level also increases and has maximum value around $\phi_{s}=20^{\circ}$ as shown in the figure. Fig. 5 shows a normalized field pattern for the two half planes of $\phi_{1}=270^{\circ}, \phi_{i}=225^{\circ}$, and $2 s=1 \lambda$ in the case of four different oblique incidence angles of $\theta_{i}=30^{\circ}, 45^{\circ}, 60^{\circ}$, and $90^{\circ}$. As $\theta_{i}$ decreases, the level of total field pattern increases, as shown in the figure. Normalized backscattered field of $E_{z}^{s} / E_{z}^{i}$ versus $\phi_{s}$ is shown in Fig. 6 for different gaps between two parallel edges of conducting half planes of $\phi_{1}=270^{\circ}$ in the case of oblique incidence $\theta_{i}=45^{\circ}$.

\section{CONCLUSION}

The behavior of TM wave scattering by a pair of infinitely long parallel edges of conducting half planes is examined in this paper. The radial mode-matching technique is used to obtain the scattered field in a series form. This new formulation analyzes scattering from a pair of half-planes geometry for oblique (skew) as well as perpendicular incidences. The diffraction coefficient for double edges is derived and formulated from the scattered field and presented in series form. The accuracy of the present method is checked with existing solutions of a planar slit and right-angle sharp wedge slit, which are special cases of the general geometry of a pair of conducting half planes.

\section{REFERENCES}

[1] J. B. Keller, "Geometrical theory of diffraction," J. Opt. Soc. Amer., vol. 52, pp. 116-130, 1962.

[2] R. G. Kouyoumjian and P. H. Pathak, "A uniform geometrical theory of diffraction for an edge in a perfectly conducting surface," Proc. IEEE, vol. 62, pp. 1448-1461, Nov. 1974.

[3] R. Tiberio and R. G. Kouyoumjian, "A analysis of diffraction at edges illuminated by transition region fields," Radio Sci., vol. 17, pp. 323-336, 1982.

[4] _ "Calculation of the high-frequency diffraction by two nearby edges illuminated at grazing incidence," IEEE Trans. Antennas Propagat., vol. AP-32, pp. 1186-1196, Nov. 1984

[5] A. Michaeli, "A new asymptotic high-frequency analysis of electromagnetic scattering by a pair of parallel wedges: Closed form results," Radio Sci., vol. 20, pp. 1537-1548, 1985.

[6] "A hybrid asymptotic solution for the scattering by a pair of parallel perfectly conducting wedges," IEEE Trans. Antennas Propagat., vol. 38, pp. 664-667, May 1990.

[7] M. Schneider and R. J. Luebbers, "A general, uniform double wedge diffraction coefficient," IEEE Trans. Antennas Propagat., vol. 39, pp. 8-14, Jan. 1991

[8] L. P. Ivrissimtzis and R. J. Marhefka, "Double diffraction at a coplanar skewed edge configuration," Radio Sci., vol. 26, pp. 821-830, 1991.

[9] _ "A uniform ray approximation of the scattering by polyhedral structures including higher terms," IEEE Trans. Antennas Propagat., vol. 40, pp. 1302-1312, Nov. 1992.

[10] J. B. Keller, "Diffraction by an aperture," J. Appl. Phys., vol. 28, no. 4, pp. 426-444, Apr. 1957.

[11] W. Pauli, "On asympotic series for functions in the theory of diffraction of light," Phys. Rev., vol. 54, pp. 924-931, 1996.

[12] J.-W. Yu and N.-H. Myung, "Diffraction by a dielectric-loaded half plane with eccentric edge," Inst. Elect. Eng. Electron. Lett., vol. 32, no. 21, pp. 1976-1978, Oct. 1996. 


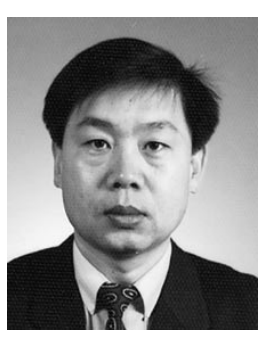

Noh-Hoon Myung was born in Seoul, Korea, in 1953. He received the B.S. degree in electrical engineering from the Seoul National University, Kores, in 1976, and the M.S. and Ph.D. degrees from The Ohio State University, Columbus, in 1982 and 1986, respectively.

From 1981 to 1986 he was a Research Member at the ElectroScience Laboratory, The Ohio State University, in the area of electromagnetic wave scattering and propagation before he joined the Electrical Engineering Department, KAIST (Korea Advanced Institute of Science and Technology), as an Assistant Professor. He was also a member of the research staff at the National Highway Traffic and Safety Administration (NHTSA), OH, where he constructed a groundbased optical vehicle tracking system. His current research areas include wave scattering and propagation analyses, RCS analysis, antenna and radar system design, mobile and satellite communications, and electromagnetic interference/electromagnetic compatibility (EMI/EMC).

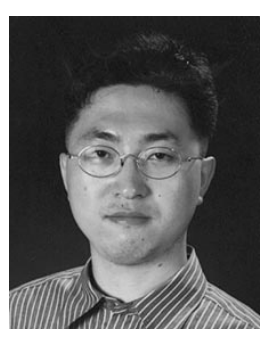

Jong-Won Yu was born in Yeosu, Korea, in 1970. He received the B.S., M.S., and Ph.D. degrees in electrical engineering from the Korea Advanced Institute of Science and Technology (KAIST), Taejon, Korea, in 1992, 1994, and 1998, respectively.

In 1998, he joined the Code Division Multiple Access (CDMA) division of System LSI Business, SamSung Electronics Co., Ltd, where he is Senior Engineer of the Field Application Group of CDMA T/F. He is researching the CDMA wireless transceiver for PCS and IMT2000 applications. His research interests are in wave propagation and scattering and mobile communication. 